\title{
OURICURI
}

\section{ACIDENTES POR SERPENTES PEÇONHENTAS (Squamata; Reptilia) EM INDÍGENAS NO BRASIL}

\author{
Aline Dantas de FREITAS ${ }^{*}$; Caroline Alves FEITOSA ${ }^{2}$, Artur Dias Lima ${ }^{3}$ \\ ${ }^{1}$ Graduanda do curso de Biomedicina na Escola Bahiana de Medicina e Saúde Pública (EBMSP). Rua \\ Silveira Martins, $n^{\circ}$ 3386, Cabula, Salvador, Bahia. CEP: 41150-100. *Autora correspondente. E- mail: \\ alinefreitas14.1@bahiana.edu.br. \\ 2Professora na Escola Bahiana de Medicina e Saúde Pública. Salvador- BA \\ ${ }^{3}$ Professor na Escola Bahiana de Medicina e Saúde Pública (EBMSP); Professor da Universidade do Estado \\ da Bahia (UNEB), Salvador, Bahia \\ Recebido: 10.12.2018 Aceito: 17.06.2019 \\ http://doi.org/10.29327/ouricuri.v9.i1.a2
}

Resumo: O objetivo deste estudo, foi analisar a frequência e distribuição dos acidentes por serpentes peçonhentas em populações indígenas no Brasil. Foi realizado um delineamento ecológico espacial e temporal, descritivo, de caráter exploratório, com abordagem quantitativa no Brasil, durante o período de 2007 - 2016, com base nos dados disponíveis no Sistema de Informações de Agravos de Notificação (SINAN). Os resultados demonstraram 8.707 casos notificados, com predomínio nas regiões Norte e Centro-Oeste, e com predomínio de acidentes provocados pelo gênero Bothrops (86\%). Quanto a gravidade, a maioria dos acidentes foram classificadas como leves, a maior frequência de acidentes ocorreu entre as faixas etárias de 20-39 anos para ambos os sexos, e a ocorrência desses acidentes é consideravelmente maior em todas as regiões com os homens $69 \%$ quando comparados as mulheres $31 \%$. Foi possível estabelecer comparações através da frequência dos acidentes ofídicos em populações indígenas entre as regiões do Brasil quanto aos gêneros das serpentes envolvidas nos acidentes, assim como, relacionar com as fitofisionomias regionais e discutir sobre medidas profiláticas. Esse estudo apresenta uma significativa contribuição para o planejamento e execução de medidas voltadas para vigilância em saúde e atendimento das necessidades específicas das populações indígenas no Brasil.

Palavras-Chave: Ofidismo; Saúde pública; Doenças tropicais negligenciadas.

\section{ACCIDENTS BY VENOMOUS SNAKES (Squamata; Reptilia) IN INDIGENOUS POPULATIONS IN BRAZIL}

Abstract: The objective of this study was to analyze the frequency and distribution of accidents by venomous snakes in indigenous populations in Brazil. An exploratory, descriptive, spatial and temporal ecological design with quantitative approach was carried out in Brazil during the period 2007-2016, based on the data available in the SINAN Information System. The results showed that there were 8,707 reported cases, with predominance in the North and Central-West Regions, there was a predominance of accidents caused by the Bothrops genus (86\%). As for severity, most of the accidents were classified as mild, the highest frequency of accidents occurred between the age groups of 20-39 years for both sexes, the occurrence of such accidents is considerably higher in all regions with men $69 \%$ when compared to women $31 \%$.It was possible to establish comparisons through the frequency of snakebite accidents in indigenous populations among the Regions of Brazil regarding the genera of snakes involved in accidents, as well as to relate to regional phytophysiognomies and to discuss prophylactic measures. This study presents a significant 
contribution to the planning and execution of measures aimed at health surveillance and meeting the specific needs of indigenous populations in Brazil.

Key words: Snakes; Public health; Neglected diseases.

\section{ACCIDENTES POR SERPIENTES PEÇONHENTAS (Squamata, Reptilia) EN INDÍGENAS EN BRASIL}

Resumen: El objetivo de este estúdio, fue analizar la frecuencia y distribución de los accidentes por serpientes peçonhen-tas en poblaciones indígenas en Brasil.Se realizó un delineamiento ecológico espacial y temporal, descriptivo, de carácter exploratorio, con abordaje cuantitativo en Brasil durante el período de 2007 a 2016, con base en los datos disponibles en el Sistema de Información de Agravios de Notificación (SINAN).Los resultados demostraron 8.707 casos notificados, con predominio en las Regiones Norte y Centro-Oeste, hubo predominio de accidentes provocados por el género bothrops (86\%). En cuanto a la gravedad, la mayoría de los accidentes se clasificaron como leves, la mayor frecuencia de accidentes ocurrió entre las edades de 20 a 39 años para ambos sexos, la ocurrencia de estos accidentes es considerablemente mayor en todas las regiones con los hombres el $69 \%$ en comparación con las mujeres un 31\%.Fue posible establecer comparaciones a través de la frecuencia de los accidentes ofídicos en poblaciones indígenas entre las Regiones de Brasil en cuanto a los géneros de las serpientes involucradas en los accidentes, así como, relacionarse con las fitofisionomías regionales y discutir sobre medidas profilácticas. Este estudio presenta una significativa contribución para la planificación y ejecución de medidas dirigidas a la vigilancia en salud y atención de las necesidades específicas de las poblaciones indígenas en Brasil.

Palabras clave: Mordedura de serpiente; Salud pública; Enfermedades tropicales olvidadas.

\section{INTRODUÇÃO}

No Brasil, as populações indígenas residentes que se declaram ou que se consideram indígenas de acordo com suas tradições, costumes e antepassados são estimadas em 896.917, pertencentes a 305 etnias distintas, distribuídas em todas as regiões. A região Norte e Centro-Oeste possui a maior concentração de indígenas que ainda permanece em terras oficialmente reconhecidas, enquanto a região Sul, apresenta o menor número de habitantes. Em contrapartida, nas regiões Sudeste e Nordeste os indígenas são mais representativos fora das terras tradicionalmente ocupadas por esta população (Instituto Brasilieiro de Geografia e Estatística IBGE, 2010).

Tanto no Brasil, como em outras partes do mundo, muitas populações indígenas ainda vivem em áreas rurais de difícil acesso. Apresentam crenças e costumes particulares na forma como se relacionam com o meio ambiente, inserindo-se de diferentes maneiras na sociedade, constituindo um dos segmentos mais desfavorecidos do ponto de vista socioeconômico, educacional e habitacional. Tendo os resultados mais alarmantes nos indicadores de saúde, como apresentam os censos que avaliam as condições de vida da população brasileira (Instituto Brasilieiro de Geografia e Estatística - IBGE, 2010).

Atualmente, os acidentes ofídicos estão inseridos na lista das doenças tropicais negligenciadas, que na maioria dos casos, acometem as populações que vivem em áreas rurais onde predomina a atividade agropecuária. Constituindo um sério problema para a saúde pública, 
pela frequência e gravidade apresentada. Relata-se que a maior parte dos casos registrados de acidentes ofídicos em todo o Brasil, são provocados por serpentes pertencentes aos gêneros Bothrops, Crotalus, Lachesis e Micrurus, e entre os anos de 2001 a 2012 os registros são de aproximadamente 326.481 mil casos, com alta incidência do gênero Bothrops (Chippaux, 2015).

No entanto, apesar do conhecimento sobre os acidentes por serpentes peçonhentas envolvendo indígenas serem de grande importância para saúde pública, no Brasil, existe uma escassez de pesquisas que trazem dados significativos sobre este tema. Certamente, conhecer as razões e obter essas informações são fundamentais para compreender o perfil epidemiológico da saúde dos indígenas no Brasil que ainda é pouco conhecido devido à escassez de investigações, no sentido de possibilitar estudos sobre a situação da saúde indígena (Ferreira et al., 2011).

Em vista disso, estudos sobre a incidência dos acidentes ofídicos através de levantamentos de dados, são de extrema relevância para a comunidade científica e para os profissionais de saúde, contribuindo para o entendimento desse agravo, possibilitando mais estudos sobre a situação de saúde desses grupos e na definição de medidas de prevenção destes acidentes. Assim, esse estudo objetivou divulgar informações importantes, que são pouco analisadas, acerca dos acidentes ofídicos que acometem indígenas no Brasil. Sobretudo, apresentando para sociedade brasileira um retrato da dinâmica dos povos indígenas que ainda permanecem com pouca visibilidade sociodemográfica. Deste modo, contribuindo para o planejamento das políticas públicas voltadas para os povos indígenas e possibilitando que ocorra importantes ações de vigilância em saúde.

\section{MATERIAL E MÉTODOS}

O presente estudo é de delineamento ecológico espacial e temporal, descritivo, de caráter exploratório, com abordagem quantitativa. Foi realizado utilizando dados secundários obtidos no Sistema de Informação de Agravos de Notificação (SINAN), através da ferramenta de tabulação de dados do DATASUS (TABNET). Os dados referiam-se a todos novos casos de acidentes ofídicos em populações indígenas notificados no Brasil no período de 2007 a 2016. As variáveis selecionadas para análise: Região de ocorrência, identificação da serpente por gênero, números de casos de acidentes por serpentes peçonhentas, ano do acidente, mês de ocorrência, dados da vítima (faixa etária e sexo), classificação final dos acidentes (leve, moderado ou grave) e evolução do caso (óbito pelo agravo notificado). Além disso, foram extraídos dados demográficos do Instituto Brasileiro de Geografia e Estatística (IBGE) e acesso no órgão indigenista Fundação Nacional do Índio (FUNAI) utilizando como base informações mais relevantes sobre o assunto. A partir de uma análise descritiva, os dados foram extraídos e analisados para geração de gráficos e tabelas.

Acrescenta-se também, que o presente estudo seguiu as normas dispostas na Resolução 466/12 do Conselho Nacional de Ética em Pesquisa e por se tratar de um estudo que utiliza dados secundários de domínio público, não há necessidade de autorização pelo comitê de ética em pesquisa. 


\section{RESULTADOS}

No Brasil, durante o período de 2007 a 2016 foram notificados 8.707 casos de acidentes ofídicos em populações indígenas no Sistema de Informação de Agravos de Notificação (SINAN). Esses acidentes, correspondem a $4 \%$ do total de casos registrados no mesmo período, quando comparado com a população em geral, independentemente da raça/cor. Houve aumento da incidência de 687 casos ocorridos em 2007, para 963 casos ocorridos em 2016, sendo que o ano de 2015 apresentou o maior número de casos notificados quando somados os acidentes de todas as regiões de estudo.

Analisando a serie temporal destes acidentes por região de notificação, através dos coeficientes anuais de incidência para cada 100.000 habitantes, observou-se que no Brasil de maneira geral, houve um aumento do número de notificações por acidentes ofídicos. Porém, com exceção das regiões Norte e Centro-Oeste, as outras regiões permaneceram estagnadas ou houve redução (Figura 1). A distribuição dos acidentes de acordo com o gênero da serpente envolvida, indica um predomínio considerável do acidente provocado por Bothrops (86\%), Lachesis (7\%), Crotalus (6\%) e Micrurus (1\%) (Figura 2). Ao analisar a distribuição mensal desses acidentes ofídicos, percebe-se que houve ocorrência de casos durante todos os meses para todos os gêneros (Figuras 3 e 4). Assim como, significativo aumento de ocorrência de casos no mês de março, provocados pelos gêneros Crotalus (Figura 3A) e Bothrops (Figura 3B), e declínio dessas ocorrências entre o mês de agosto e setembro. O gênero Lachesis, apresentou maior número de ocorrência de casos de acidentes no mês de maio, e declínio entre os meses de agosto a outubro (Figura 4A). Já para o gênero Micrurus, houve mais ocorrências no mês de abril e redução de ocorrências de casos entre os meses de outubro a novembro (Figura 4B).

Em relação às características dos indivíduos acometidos, a maior frequência de acidentes ofídicos ocorreu entre as faixas etárias de 20-59 anos, para ambos os sexos (Figura 5). Observase, ocorrência de acidentes em todas as regiões e prevalência do sexo masculino. A relação destes, é equivalente a $69 \%$ para o sexo masculino e $31 \%$ para acidentes com o sexo feminino (Figura 6). Quanto à severidade, verificou-se que nos acidentes provocados por serpentes do gênero Bothrops, os casos leves foram predominantes, com 3.161 registrados (46\%), seguidos de 2.745 moderados (40\%) e 665 graves (10\%). Para o gênero Crotalus, foram observados 199 (39\%) dos casos classificados como leves, 198 (39\%) moderados e 84 (17\%) graves. Já para o gênero Lachesis, 160 (27\%) dos casos classificados como leves, 337 (57\%) moderados e 61 (10\%) graves. Observa- se ainda, que 374 (5\%) das notificações não foram preenchidas corretamente quanto ao gênero da serpente envolvida nos acidentes, sendo, portanto, classificadas como ignorado/branco (Figura 7). No período analisado, foi registrado 78 óbitos provocados por acidentes ofídicos. De maneira geral, a maioria dos casos fatais ocorreram em indivíduos idosos com faixa etária entre 6079 anos ou superior (Tabela 1). 


$\begin{array}{ccccccccc}2008 & 2009 & 2010 & 2011 & 2012 & 2013 & 2014 & 2015 & 2016 \\ 117 & 129,2 & 114,9 & 156,6 & 182,9 & 180,8 & 184,1 & 180 & 194,3 \\ 47,7 & 52,4 & 61,4 & 69,2 & 58,9 & 50,3 & 54,1 & 53,3 & 32,7 \\ 32,3 & 30,3 & 36,3 & 35,3 & 20,2 & 26,2 & 30,3 & 38,3 & 17,1 \\ 30,5 & 53,3 & 41,9 & 49,5 & 35,5 & 30,5 & 44,4 & 47 & 33 \\ 110,2 & 116,4 & 78,1 & 111,6 & 115 & 92 & 103,9 & 135,3 & 124,1\end{array}$

Figura 1. Coeficientes de incidência anual (por 100.000 habitantes) dos acidentes por região. Brasil. Fonte: SINAN-DATASUS/Ministério da Saúde, 2016.

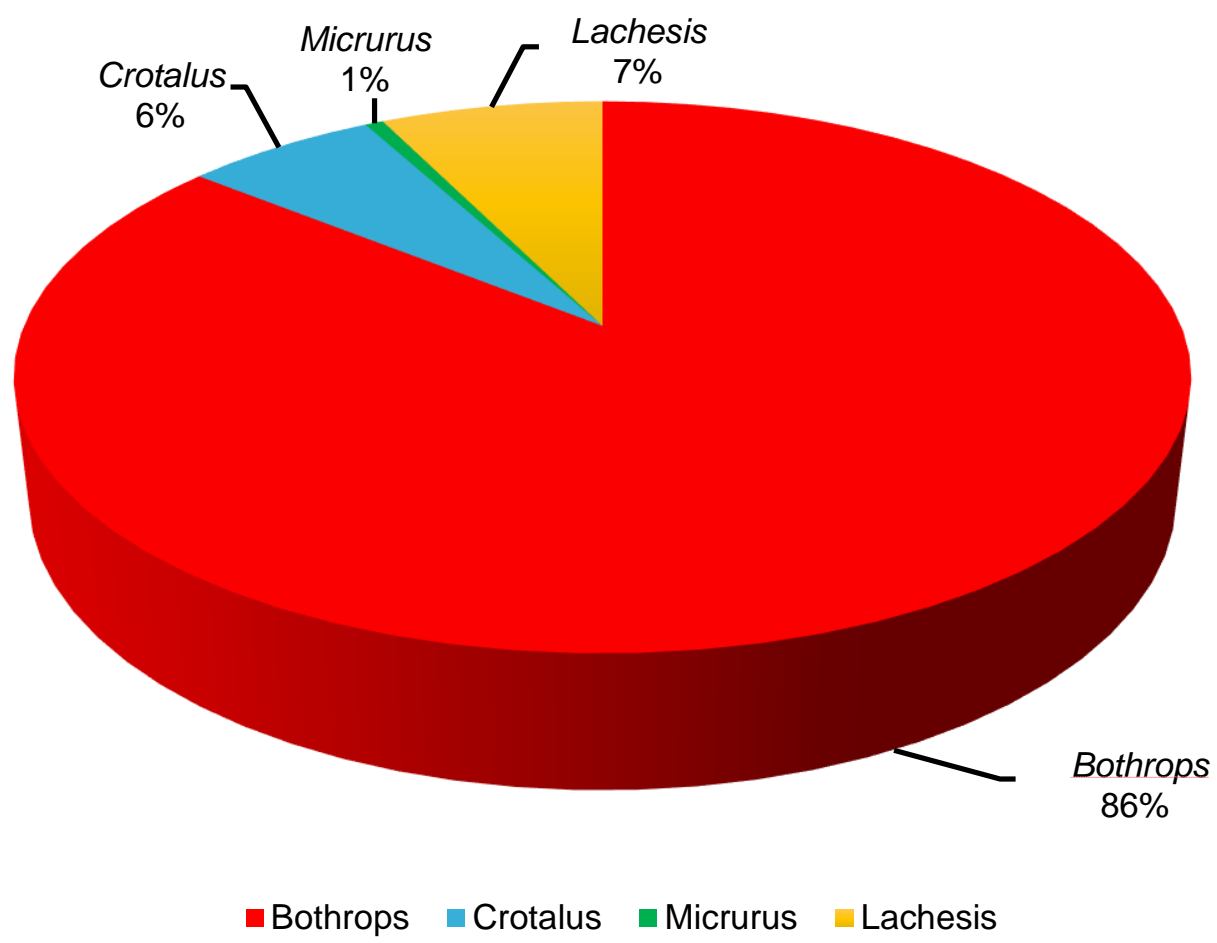

Figura 2. Distribuição dos acidentes ofídicos segundo o gênero da serpente. Brasil, 2007 a 2016. Fonte: SINAN-DATASUS/Ministério da Saúde, 2016. 
A 70

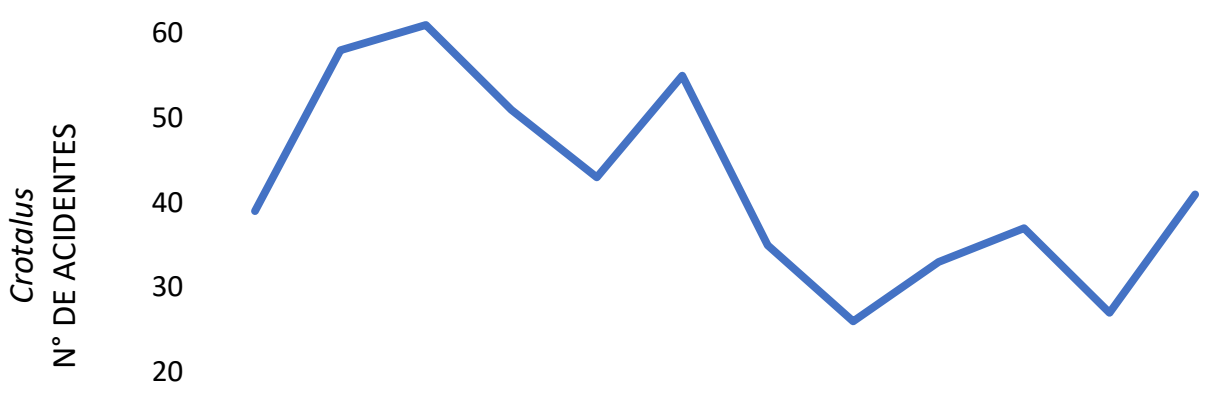

10

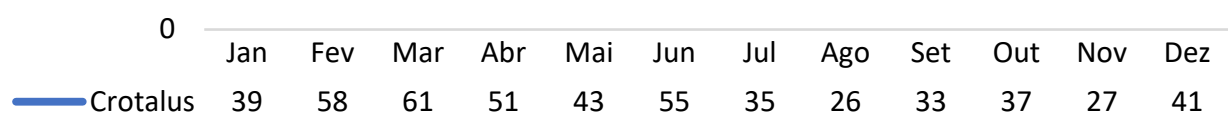

MÊS DE OCORRÊNCIA

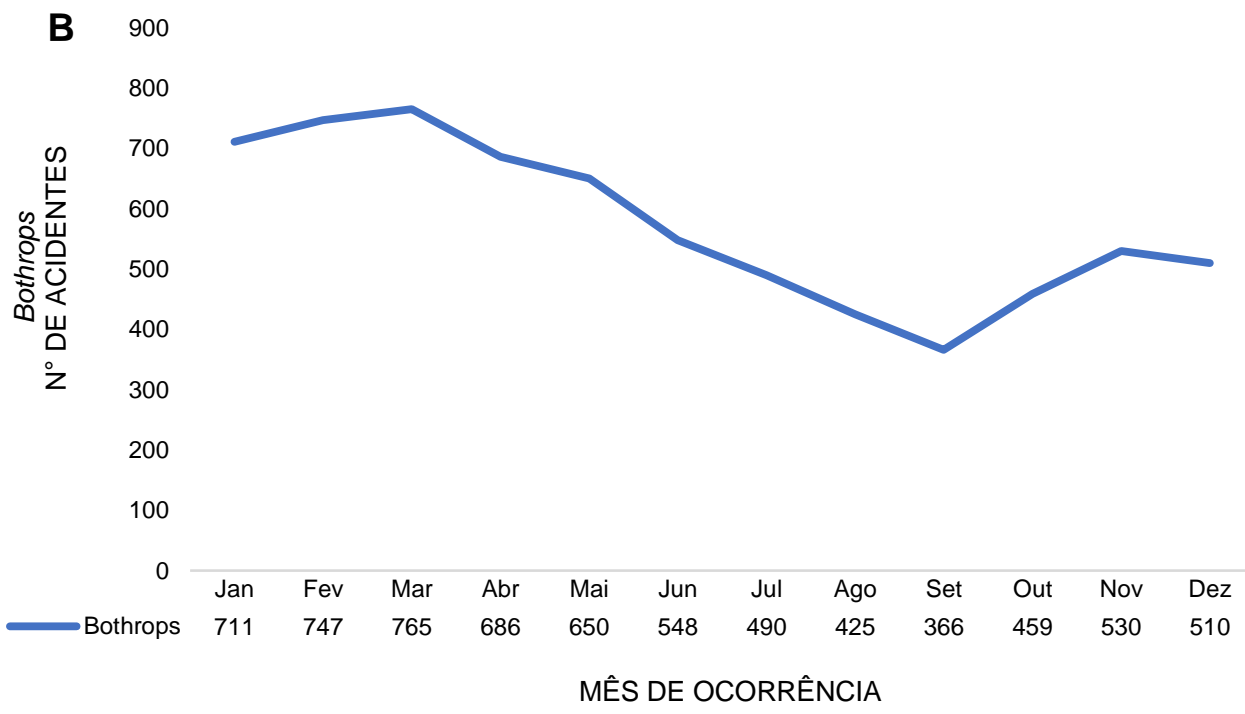

Figura 3. Distribuição mensal dos acidentes ofídicos no Brasil, causados por Crotalus (A) e Bothrops (B), de 2007 a 2016. Fonte: SINAN-DATASUS/Ministério da Saúde, 2016. 

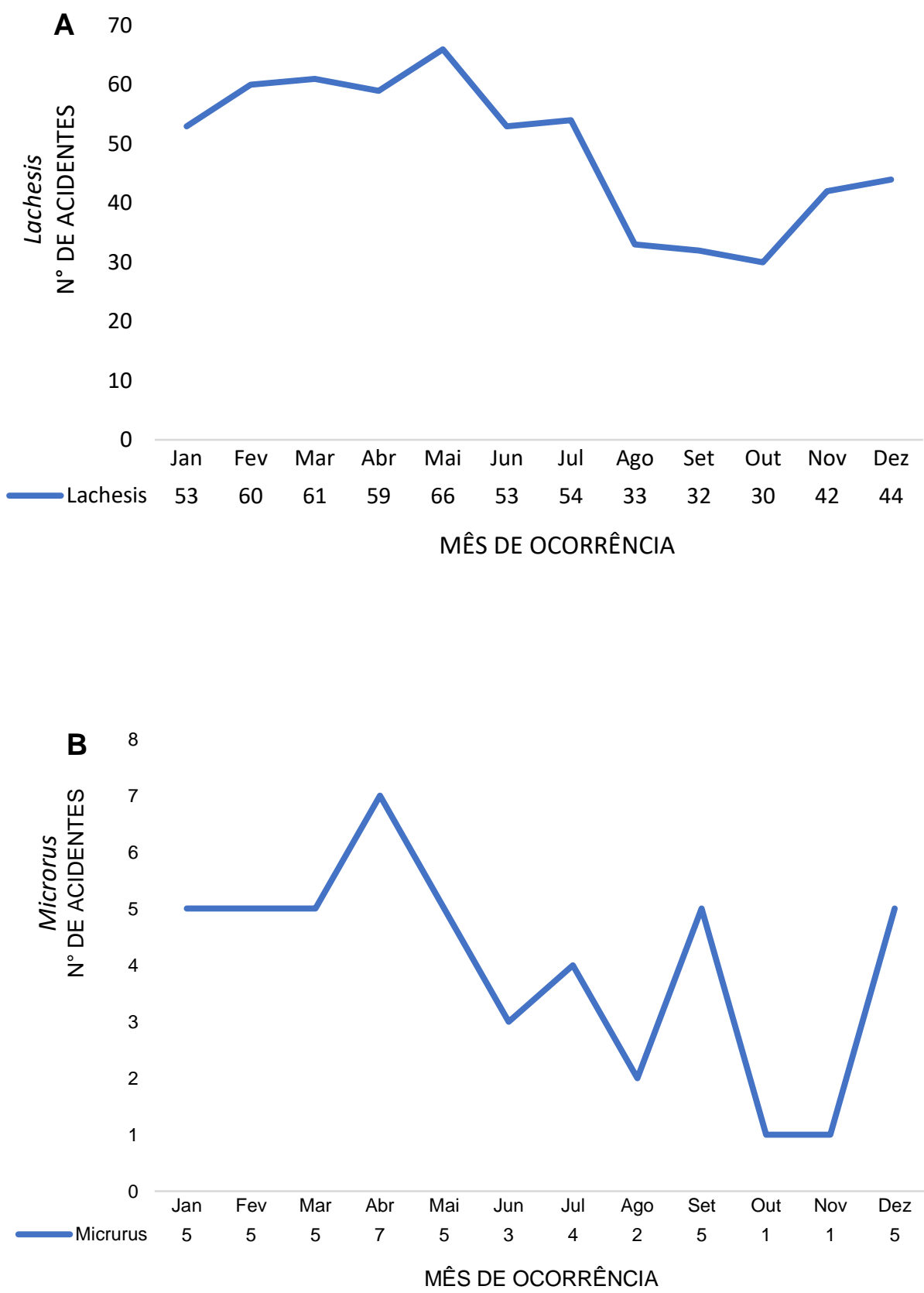

Figura 4. Distribuição mensal dos acidentes ofídicos no Brasil, causados por Lachesis (A) e Micrurus (B), de 2007 a 2016. Fonte: SINAN-DATASUS/Ministério da Saúde, 2016. 
2500

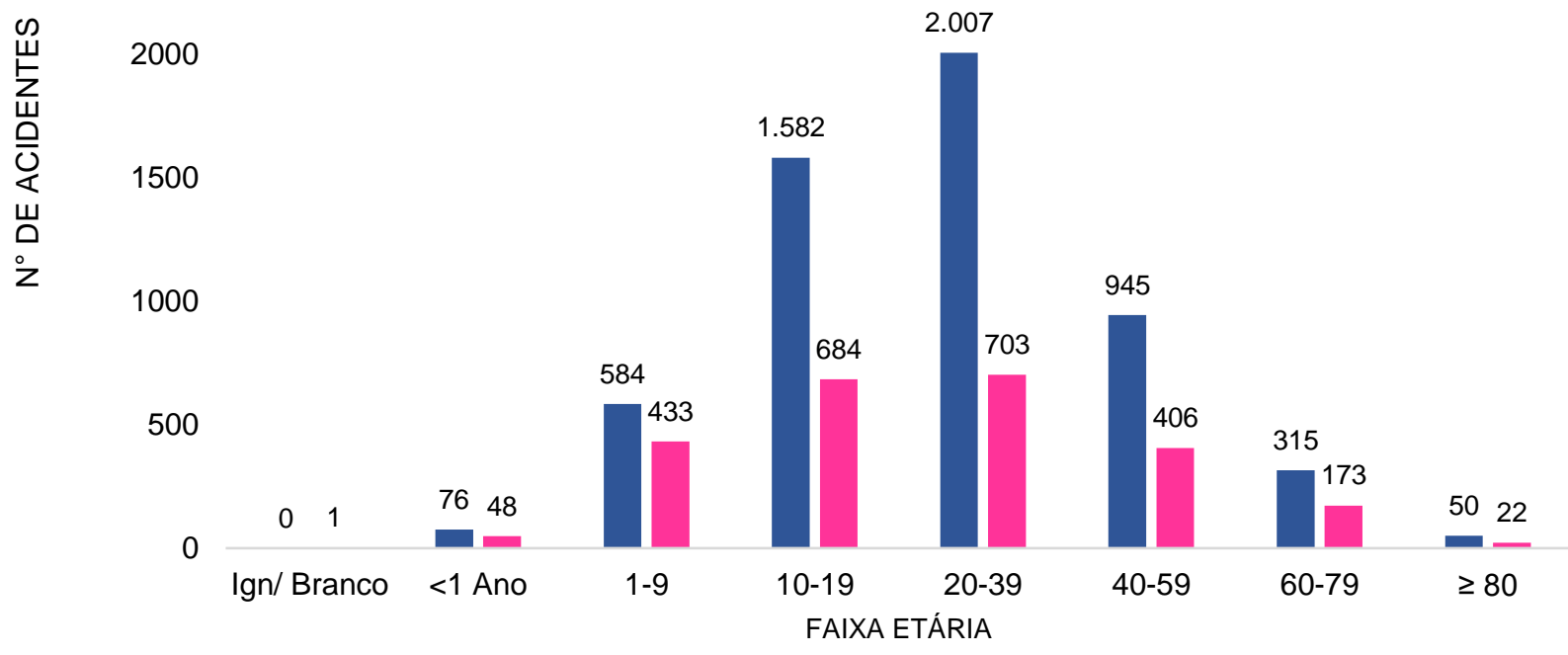

- Masculino Feminino

Figura 5. Distribuição por faixa etária. Brasil, 2007 a 2016. Fonte: SINAN-DATASUS/Ministério da Saúde, 2016.

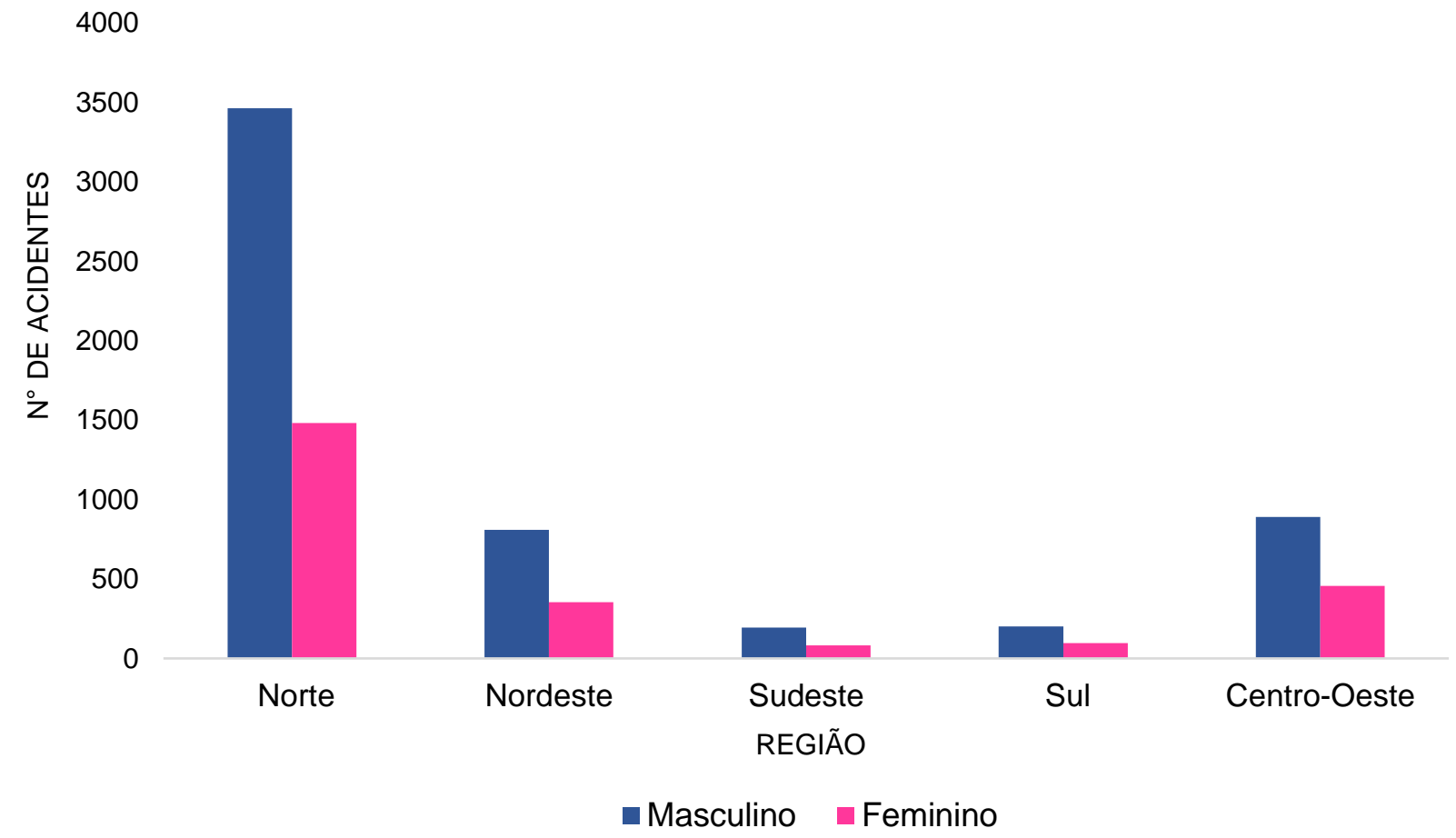

Figura 6. Distribuição por macrorregião de notificação e sexo, Brasil, 2007 a 2016. Fonte: SINANDATASUS/Ministério da Saúde, 2016. 


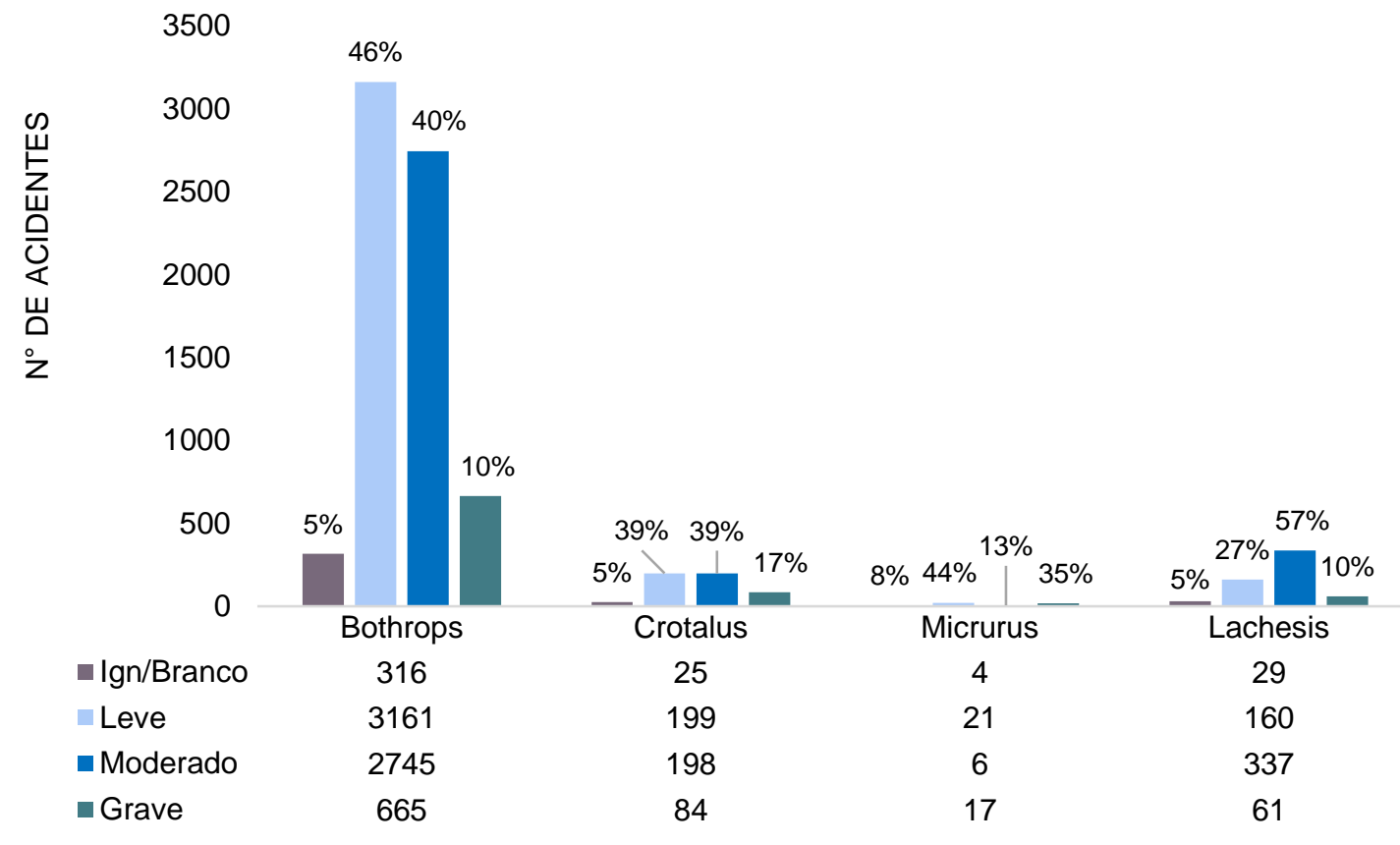

Figura 7. Classificação final dos acidentes ofídicos, segundo gênero envolvido. Brasil, 2007 a 2016. Fonte: SINAN-DATASUS/Ministério da Saúde, 2016.

Tabela 1. Distribuição por faixa etária, do número de casos, óbitos e letalidade dos acidentes ofídicos. Brasil, 2007 a 2016.

\begin{tabular}{lccc}
\hline Faixa etária & $N^{\circ}$ Casos & $N^{\circ}$ Óbitos & Letalidade (\%) \\
\hline$<1$ ano & 124 & 1 & 0,8 \\
$1-9$ anos & 1.017 & 14 & 1,4 \\
$10-19$ anos & 2.226 & 17 & 0,8 \\
$20-39$ anos & 2.710 & 16 & 0,6 \\
$40-59$ anos & 1.350 & 13 & 1,0 \\
$60-79$ anos & 488 & 13 & 2,7 \\
$\geq 80$ anos & 72 & 4 & 5,6 \\
Ign/Branco & 858 & - & - \\
Total & 8.885 & 78 & 0,9 \\
\hline
\end{tabular}




\section{DISCUSSÃO}

A análise da frequência dos acidentes por serpentes peçonhentas em populações indígenas no Brasil, de acordo com dados secundários obtidos no Sistema de Informação de Agravos de Notificação (SINAN), permitiu identificar no presente estudo que entre todas as regiões avaliadas, a incidência de acidentes ofídicos ocorre com maior predomínio nas regiões Norte e Centro-Oeste. O aumento de notificações nessas regiões, pode ser atribuído ao fato de a maior parte das populações indígenas permanecerem habitando em áreas rurais, caracterizando assim uma área em que a população está mais exposta a atividades que podem propiciar o risco desse tipo de acidente. Ao contrário das demais regiões analisadas, que apresenta menor número de habitantes indígenas ou esses são mais representativos fora das terras tradicionalmente ocupadas por esta população, como ocorre na região Nordeste (Araújo et al., 2003).

Todas as regiões avaliadas apresentam fatores de risco e exposição para ocorrência dos acidentes ofídicos, devido a variação em termos de aspectos climáticos e vegetação. Na região Norte, o clima é equatorial e a vegetação é a floresta amazônica, apresentando algumas manchas de cerrado. No Centro-Oeste, predomina o clima tropical e a vegetação do tipo cerrado. No Nordeste o clima é tropical, semiárido e a vegetação do tipo caatinga, com matas tropicais. No Sudeste o clima é tropical e a vegetação é a Mata Atlântica, já no Sul o clima é subtropical e predomina a vegetação de campos (CHIPPAUX, 2015). Os gêneros das serpentes peçonhentas Bothrops, Lachesis e Micrurus, vivem exclusivamente em áreas florestais, enquanto o gênero Crotalus é típico de áreas abertas, e devido ao desmatamento tem aumentado o número de ocorrências de casos de acidentes ofídicos (Cardoso et al., 2003).

Deve-se considerar também que, embora haja notificações para todas as serpentes peçonhentas em todas as regiões do Brasil, houve predomínio considerável de acidentes ofídicos provocados por Bothrops. Esse gênero, apresenta uma ampla distribuição geográfica e está presente em zonas rurais e urbanas. Preferencialmente ambientes úmidos, como áreas florestadas, margens de rios e locais onde há facilidade para proliferação de roedores. Esses dados, estão de acordo com as estatísticas nacionais nas quais verifica-se o predomínio do acidente botrópico (BRASIL, 2009).

Não houve significativa variação regional na distribuição dos acidentes por Micrurus, que foram $1 \%$ das notificações em todas as regiões analisadas. Essas observações reforçam os dados já existentes na literatura, onde foi relatado que acidentes pelo gênero Micrurus, raramente causam acidentes em seres humanos (Casais-e-silva e Brazil, 2009). Evidenciando que no Brasil, algumas espécies de Micrurus, são raras ou pouco conhecidas e distribuídas em áreas geográficas restritas (Cardoso et al., 2009).

Quanto à sazonalidade, é possível verificar que nos meses com temperaturas mais elevadas, referentes ao verão e ao final da primavera, ocorreram os maiores números de casos de acidentes para os gêneros Bothrops, Crotalus, Lachesis e Micrurus. Justamente, porque durante os 
períodos mais frios e secos há uma queda no metabolismo das serpentes, e provavelmente menor disponibilidade de presas para sua alimentação (Moreira, 2014). Outro fator importante a ser considerado, é o aumento das atividades extrativistas em áreas rurais, principalmente pelas populações indígenas nos períodos citados de maior ocorrência de acidentes ofídicos (Waldez e Vogt, 2009).

O estudo mostrou que indígenas com idade entre 20-39 anos, representam as principais vítimas dos acidentes ofídicos, a baixa frequência em indivíduos acima de 60 anos pode indicar menor participação nos trabalhos de campo. Dentre os indivíduos envolvidos, observou-se predomínio do sexo masculino, independente da região. Esses dados, corroboram com dados apresentados em outro estudo, que justifica o predomínio do sexo masculino pelo envolvimento com atividades agropecuárias para sustento das famílias, muitas vezes não fazem uso dos equipamentos de proteção individual, expondo-se ao risco dos acidentes ofídicos (Graciano et al., 2013).

Quanto à classificação da gravidade dos casos, houve predomínio de ocorrência de acidentes provocados por serpentes do gênero Bothrops classificados como leves. O tempo entre o acidente e o atendimento prestado as vítimas, é fundamental para reduzir as chances de aumentar a gravidade dos acidentes (Lima et al., 2009). Estudos como o de Chippaux (2015), estão de acordo com as taxas de letalidade encontradas neste estudo, onde a letalidade geral para o Brasil é baixa, em torno de $1 \%$, ou seja, apenas um a cada 100 acidentes que ocorrem com serpentes peçonhentas resultam em óbito. Contudo, vale ressaltar que os idosos constituem o grupo mais vulnerável por apresentarem maiores índices de letalidade que chega a ser cinco vezes maior do que em outras faixas etárias. Estudos mostram que idosos maiores de 60 anos apresentam maior probabilidade de apresentar necrose na região da picada, justificando assim, uma taxa de letalidade mais alta nessa faixa etária (Ribeiro et al., 2008).

Os acidentes provocados por serpentes peçonhentas podem ocorrer por diferentes causas, principalmente em populações indígenas. Uma vez que, além das atividades agropecuárias, os indígenas em sua relação direta com ambientes naturais, muitas vezes sem a utilização dos equipamentos de proteção individual, estão expostos diretamente ao risco de acidentes ofídicos. Por isso, medidas de prevenção devem ser adotadas: utilização de calçados fechados ou botas de cano alto, usar luvas de couro e evitar acúmulo de entulhos próximos das residências que possam servir de abrigo para serpentes peçonhentas e roedores. Em caso de ocorrência do acidente, é importante: lavar a região da picada apenas com água e sabão, levar a vítima para atendimento médico o mais rápido possível, não fazer torniquete ou garrote no membro picado, pois isso poderá agravar o acidente, aumentando a concentração do veneno no local (Bernarde, 2015).

Diante disso, é de suma importância que as populações indígenas sejam cada vez mais inseridas em espaços de participação de saúde visando compreender sobre adoção de medidas profiláticas em casos de acidentes, assim como, treinamento dos profissionais de saúde para 
preenchimento completo das fichas de notificações contribuindo para o entendimento desse agravo e possibilitando que ocorra importantes ações de vigilância em saúde e na definição de medidas de prevenção destes acidentes. O presente estudo apresentou limitações, relacionadas com a perda de qualidade dos dados disponíveis do SINAN devido a um elevado número de notificações não preenchidas corretamente, sendo reportadas como ignoradas/Branco com relação a faixa etária, classificação final dos casos e identificação do gênero da serpente. Ressalta-se, que o SINAN não oferece ferramentas para diferenciação das espécies de cada gênero durante a captação de dados, todavia, no Brasil, encontra-se uma expressiva diversidade de espécies de interesses médicos e a correta identificação no momento do atendimento contribuirá de forma significativa na adoção do tratamento adequado (PARISE, 2016).

Essas limitações podem ainda ser agravadas se relacionadas aos conhecimentos e práticas tradicionais indígenas, que vivem longe dos centros urbanos, e por vezes, pela distância ou desconhecimento da importância do tratamento recusam atendimento dos serviços de saúde conduzindo os próprios cuidados sem o acompanhamento médico, consequentemente, não formalizando a notificação, gerando um possível índice abaixo da realidade. É possível, ainda, a ocorrência de subnotificações ao uso dos dados do SINAN. Estudo de Fiszon e colaboradores (2008), demonstra que a capacidade de obtenção de dados pelo SINAN tem aumentado ao longo dos anos. No entanto, no que se refere aos casos de acidentes ofídicos as perdas de registros ainda são grandes.

\section{CONSIDERAÇÕES FINAIS}

Apesar das limitações do presente estudo, foi possível estabelecer importantes comparações dos novos casos de acidentes ofídicos em populações indígenas notificados entre as regiões do Brasil no período de 2007 a 2016, através das variáveis selecionadas para análise. Tendo em vista os aspectos observados, esse estudo apresenta uma significativa contribuição para pesquisas relacionadas aos acidentes por serpentes peçonhentas em indígenas no Brasil, visto que, há poucos estudos recentes avaliando essa situação epidemiológica dos acidentes ofídicos ocorridos em populações indígenas.

Recomenda-se então novos estudos, que avaliem a situação epidemiológica dos acidentes por serpentes peçonhentas em indígenas no Brasil. Que possam agregar na elaboração de estratégias para melhores condições de atendimento, e que as ações que garantem direitos de cidadania para povos indígenas, atualmente realizadas pela Fundação Nacional do Índio - FUNAI, órgão indigenista oficial do Estado brasileiro, continuem sendo promovidas. Visando reduzir o número de notificações e contribuindo para o planejamento das políticas públicas, servindo como importante indicador, para proporcionar melhores condições, no sentido de atender as necessidades que são específicas desta população. 


\section{REFERÊNCIAS}

Araujo, F. A. A.; Santalucia, M.; Cabral, R. F. Epidemiologia dos acidentes por animais peçonhentos. In: Cardoso J. L. C., França, F. O. S., Wen, F. H., MALAQUE C. M. S, Haddad V. J. Animais peçonhentos no Brasil: biologia, clínica e terapêutica dos acidentes. São Paulo: Sarvier, 2003, p. 69.

Bernarde, P. S. Ofidismo no estado do Acre - BRASIL. Journal of Amazon Health Science, v. 1, n. 2, 2015. Disponível em: https://periodicos.ufac.br/index.php/ahs/article/view/303. Acesso em: 09 outubro 2018.

Brasil. Ministério da Saúde. Secretaria de Vigilância em Saúde. Guia de vigilância epidemiológica. 7. ed. Brasilia: Ministério da Saúde, 2009. 816p. Disponível em: http://bvsms.saude.gov.br/bvs/publicacoes/guia_vigilancia_epidemiologica_7ed.pdf. Acesso em: 12 abril 2018.

Cardoso, J. L. C; França, F. O. S.; Wen F. H.; Málaque, C. M. S; Haddad Jr, V. Animais peçonhentos no Brasil. Biologia, clínica e terapêutica dos acidentes. 1. ed. São Paulo: Sarvier, 2003.

Cardoso, J. L. C.; França, F. O. S.; Wen, F. H.; Málaque, C. M. S; Haddad Jr, V. Animais peçonhentos no Brasil. Biologia, clínica e terapêutica dos acidentes. 2. ed. São Paulo: Sarvier, 2009.

Casais-e-Silva, L. L.; Brazil, T. K. Acidentes elapídicos no estado da Bahia: Estudo retrospectivo dos aspectos epidemiológicos em uma serie de 14 anos (1980-1993). Gazeta Médica da Bahia, 79(16), 26-31, 2009.

Chippaux, J. P. Epidemiology of envenomations by terrestrial venomous animals in Brazil based on case reporting: From obvious facts to contingencies. Journal of Venomous Animals and Toxins Including Tropical Diseases, 21(1), 1-17, 10.1186, 2015.

Ferreira, M. E.; Matsuo, T.; Souza, R. K. Demographic characteristics and mortality among indigenous peoples in Mato Grosso do Sul State, Brazil. Cadernos de Saúde pública, 27(12), 23272339, 10.1590, 2011.

FISZON, J. T.; BOCHNER, R. Subnotificação de acidentes por animais peçonhentos registrados pelo SINAN no Estado do Rio de Janeiro no período de 2001 a 2005. Revista Brasileira de Epidemiologia, v. 11, n. 1, p. 114-127, 10.1590, 2008.

Graciano, S.; Coelho, M.; Teixeira, A.; Silva, J.; Pereira, S.; Fernandes, R. Perfil epidemiológico dos acidentes ofídicos em homens. Revista de Enfermagem Referência, III Série (10), 89-98, 10.12707, 2013.

Instituto Brasileiro de Geografia e Estatística-IBGE. (2010). Censo Demográfico 2010: característica geral dos indígenas-Resultado do Universo. Censo Demográfico 2010, 1-244. Disponível em: https://biblioteca.ibge.gov.br/visualizacao/periodicos/95/cd_2010_indigenas_universo.pdf. Acesso em: 12 abril 2018.

Lima, J. S.; Martelli Júnior, H.; Martelli, D. R. B.; Silva, M. S.; Carvalho, S. F. G.; Canela, J. R.; Bonan, P. R. F. (2009). Perfil dos acidentes ofídicos no norte do Estado de Minas Gerais, Brasil. Revista Da Sociedade Brasileira de Medicina Tropical, 42(5), 561-564, 10.1590. 2009.

Moreira, J. P. L. (2014). Incidência E Ocorrência De Ataques Ofídicos No Brasil Em 2012, 1, 18361846. Disponivel em: http://www.unifal-mg.edu.br/simgeo/system/files/anexos/João Paulo Lima Moreira.pdf. Acesso em: 24 agosto 2018

PARISE, E. V. Vigilância e monitoramento dos acidentes por animais peçonhentos no Município de Palmas, Tocantins, Brasil. Hygeia, v. 12, n. 22, p. 72-87, 2016. 
Ribeiro, L. A.; Gadia, R.; Jorge, M. T. (2008). Comparação entre a epidemiologia do acidente e a clínica do envenenamento por serpentes do gênero Bothrops, em adultos idosos e não idosos. Revista Da Sociedade Brasileira de Medicina Tropical, 41(1), 46-49. 10.1590, 2008.

Waldez, F.; Vogt, R. C. Aspectos ecológicos e epidemiológicos de acidentes ofídicos em comunidades ribeirinhas do baixo rio Purus, Amazonas, Brasil. Acta Amazonica, 39(3), 681-692. 10.1590, 2009. 